\title{
Sexual transmission of Hepatitis $C$ virus between HIV infected subjects and their main heterosexual partners; Actor Partner Interdependent Modelling
}

\author{
Abbas Alipour ${ }^{*}$, Abbas Rezaianzadeh², Jafar Hasanzadeh², Abdorreza Rajaeefard², Mohammad Ali Davarpanah ${ }^{3}$ \\ From International Symposium HIV and Emerging Infectious Diseases 2014 \\ Marseille, France. 21-23 May 2013
}

\section{Introduction}

Overall, $60-70 \%$ of the hepatitis $\mathrm{C}$ virus' (HCV) transmission routes is parenteral and in $30-40 \%$ of the cases was unknown (e.g. sexual route). Knowing of these routes in HIV infected dyads very important due to clinical and methodological reasons. Whether HCV transmits through sexual route in these dyads is controversy.

\section{Materials and methods}

Of the 1338 dyads enrolled in Behavioural Consultation Center in Shiraz, Iran in 2011, 984 couples (HIV infected subjects and their main heterosexual partners) have eligibility criteria. Random samples, 168 of 984 couples, were chosen through random generated numbers using a computer program. We used actor partner independent model (APIM) and multilevel analysis to assess multiple risk factors for $\mathrm{HCV}$ while partitioning the source of risk at the individual and couple level.

\section{Results}

Age of the index samples was $38.71 \pm 7$ years and $33.2 \pm$ 6.3 years for their main heterosexual partners; the mean duration of sexual relationship for couples was 11.9 $($ median $=8.5)$ years. Multivariate analysis showed that actor risk factor of intravenous drug using (IDU) (AOR= 13.03; 95\% CI: 3.9- 43.82) and actor cofactors of HIV positivity (AOR= 7.1; 95\% CI: $1.37-36.97)$, razor sharing $(\mathrm{AOR}=4.81 ; 95 \% \mathrm{CI}: 1.84-12.55)$, $\operatorname{sex}(\mathrm{AOR}=8.83 ; 95 \%$ CI: 3.16- 24.87), and condom use in sexual activity with main partner $(\mathrm{AOR}=0.15 ; 95 \% \mathrm{CI}$ : 0.02- 0.44$)$ were associated with actor $\mathrm{HCV}$ positivity.
Table 1 Multilevel logistic regression estimation of individual and couple level risk factors for actor anti-HCV positivity, final model, Shiraz (southern of Iran), 2011

\begin{tabular}{llll}
\hline Effects $^{\dagger}$ & $\boldsymbol{\beta}$ & SE & AOR (95\% Cl) \\
\hline Actor IDU & 2.57 & 0.62 & $13.03(3.9-43.82)$ \\
\hline Actor Razor sharing & 1.57 & 0.49 & $4.81(1.84-12.55)$ \\
\hline Actor HIV positive & 1.96 & 0.84 & $7.1(1.37-36.97)$ \\
\hline Actor male & 2.18 & 0.52 & $8.83(3.16-24.78)$ \\
\hline $\begin{array}{l}\text { No condom use in sexual activity } \\
\text { with main heterosexual partner }\end{array}$ & $\mathbf{1 . 8 9}$ & $\mathbf{0 . 9 2}$ & $\mathbf{6 . 6}(\mathbf{1 . 1}-\mathbf{3 9 . 6})$
\end{tabular}

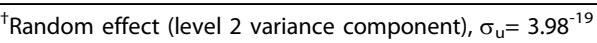

\section{Conclusion}

Health care providers need to pay special attention to sexual transmission of HCV among HIV-infected individuals and the control/preventive measures for HCV sexual transmission should be recommended.

\section{Authors' details}

'Social medicine department, Medical school, Mazandaran university of medical sciences, Sari, Iran. ${ }^{2}$ Epidemiology department, Health and Nutrition School, Shiraz university of medical sciences, Sari, Iran. ${ }^{3}$ HIV Research center, Shiraz University of medical Sciences, Sari, Iran.

Published: 23 May 2014

\section{doi:10.1186/1471-2334-14-S2-P87}

Cite this article as: Alipour et al:: Sexual transmission of Hepatitis C virus between HIV infected subjects and their main heterosexual partners; Actor Partner Interdependent Modelling. BMC Infectious Diseases 2014 14(Suppl 2):P87. 